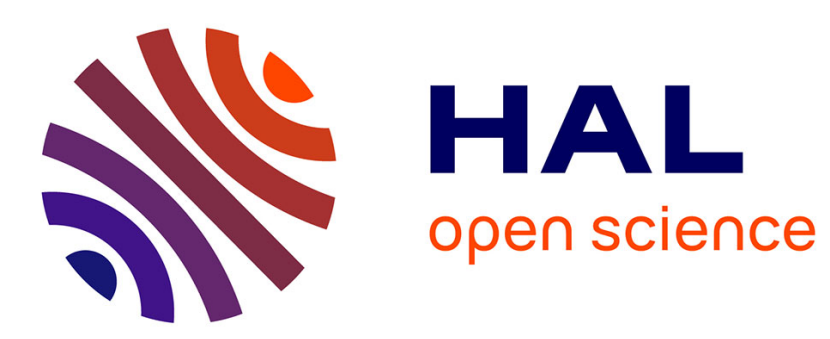

\title{
Structure cristalline de MoNb15O40F
}

Jean Galy, Sten Andersson

\section{To cite this version:}

Jean Galy, Sten Andersson. Structure cristalline de MoNb15O40F. Acta crystallographica Section B: Structural crystallography and crystal chemistry, 1968, 24, pp.1027-1031. 10.1107/S0567740868003651 . hal-00122776

\section{HAL Id: hal-00122776 https://hal.science/hal-00122776}

Submitted on 13 Jun 2013

HAL is a multi-disciplinary open access archive for the deposit and dissemination of scientific research documents, whether they are published or not. The documents may come from teaching and research institutions in France or abroad, or from public or private research centers.
L'archive ouverte pluridisciplinaire $\mathbf{H A L}$, est destinée au dépôt et à la diffusion de documents scientifiques de niveau recherche, publiés ou non, émanant des établissements d'enseignement et de recherche français ou étrangers, des laboratoires publics ou privés. 


\title{
Structure Cristalline de $\mathrm{MoNb}_{15} \mathrm{O}_{40} \mathrm{~F}$
}

\author{
PAR J. GaLY* \\ Institule of Inorganic Chemistry, Stockholm, Suede \\ et Sten ANDERsson \\ Research Institute of National Defence, Department 4, Stockholm, Suede
}

(Reçu le 4 novembre 1967)

\begin{abstract}
$\mathrm{MoNb}_{15} \mathrm{O}_{40} \mathrm{~F}$ crystallizes in the monoclinic system with lattice parameters $a=22 \cdot 29, b=3 \cdot 831, c=$ $20 \cdot 27 \AA, \beta=114^{\circ}$. The space group is $C 2$. The original structure, refined by lcast-squares methods, contains $\mathrm{ReO}_{3}$-type blocks of $\mathrm{Nb}-\mathrm{O}$ octahedra, $3 \times 5$ and infinite along $\mathrm{b}$. The blocks are joined by sharing edges and with tetrahedrally coordinated Mo atoms at the junctions of every four blocks. $\mathrm{MoNb}_{15} \mathrm{O}_{40} \mathrm{~F}$ can be represented by the structural formula:
\end{abstract}

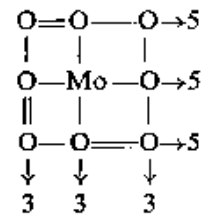

Les systèmes binaires $\mathrm{MoO}_{3}-\mathrm{NbO}_{2} \mathrm{~F}, \mathrm{NbO}_{2} \mathrm{~F}-\mathrm{Nb}_{2} \mathrm{O}_{5}$ et $\mathrm{MoO}_{3}-\mathrm{Nb}_{2} \mathrm{O}_{5}$ ont fait l'objet d'études approfondies. Si le système $\mathrm{MoO}_{3}-\mathrm{NbO}_{2} \mathrm{~F}$ ne révèle la présence d'aucune phase nouvelle, les deux autres systèmes sont par contre beaucoup plus riches.

Le système $\mathrm{NbO}_{2} \mathrm{~F}-\mathrm{Nb}_{2} \mathrm{O}_{5}$ comportc quatre composés originaux $\mathrm{Nb}_{3} \mathrm{O}_{2} \mathrm{~F}, \quad \mathrm{Nb}_{5} \mathrm{O}_{12} \mathrm{~F}, \quad \mathrm{Nb}_{17} \mathrm{O}_{42} \mathrm{~F}$ et

* Adresse permanente: Service de Chimic Minérale Structurale, Faculté des Sciences, 33-Talence, France.
$\mathrm{Nb}_{31} \mathrm{O}_{77} \mathrm{~F}$, dont la préparation et l'étude structurale sont dues à Andersson et Åström (Andersson, 1964, 1965a; Andersson \& Åström, 1964; Áström, 1966).

Dans le système $\mathrm{MoO}_{3}-\mathrm{Nb}_{2} \mathrm{O}_{5}$ deux phases ont été mises en évidence et leurs caractères cristallographiques précisés: l'une $\mathrm{Mo}_{3} \mathrm{Nb}_{14} \mathrm{O}_{44}$, de symétrie quadratique, est isotype de $\mathrm{W}_{3} \mathrm{Nb}_{14} \mathrm{O}_{44}$, l'autre $\mathrm{MoNb}_{12} \mathrm{O}_{33}$, monoclinique, est isotype de $\mathrm{WNb}_{12} \mathrm{O}_{33}$; ces composés du tungstène ont été préparés et étudiés par Roth \& Wadsley (1965). 
Dans le diagramme de phase illustré par la Fig.1, seules les phases précisées sur le plan structural ont été représentées. Les systèmes $\mathrm{MoO}_{3}-\mathrm{Nb}_{2} \mathrm{O}_{5}$ et $\mathrm{MoO}_{3}-\mathrm{Nb}_{2} \mathrm{O}_{5}-\mathrm{NbO}_{2} \mathrm{~F}$ contiennent d'autres phases originales qui toutefois ne sont pas reportées ici.

L'étude du système ternaire $\mathrm{MoO}_{3}-\mathrm{Nb}_{2} \mathrm{O}_{5}-\mathrm{NbO}_{2} \mathrm{~F}$ s'avérait extrêmement prometteuse du fait du grand nombre de combinaisons susceptibles d'être envisagées. Nous avons alors tenté de préparer le composé $\mathrm{MoNb}_{16} \mathrm{O}_{42} \mathrm{~F}_{2}$ pensant obtenir la structure quadratique de $\mathrm{W}_{3} \mathrm{Nb}_{14} \mathrm{O}_{44} ; \mathrm{W}_{3} \mathrm{Nb}_{14} \mathrm{O}_{44}$ appartient à la série $B_{n m+1} \mathrm{O}_{3 n m-(n+m)+4}($ avec $m=n=4)$ de Roth \& Wadsley (les deux variables $m$ et $n$ représentent le nombre d'octaèdres respectivement sur la largeur et la longueur des blocks de type $\mathrm{ReO}_{3}$ constituant ces structures). L'étude radiocristallographique des cristaux isolés après fusion du mélange de composition $\mathrm{MoNb}_{16} \mathrm{O}_{42} \mathrm{~F}_{2}$ révélait en fait une symétrie monoclinique; la détermination de la structure a permis de préciser la formule de ces cristaux, $\mathrm{MoNb}_{15} \mathrm{O}_{40} \mathrm{~F}$, qui correspond à un oxyfluorure double entièrement nouveau. Cet oxyfluorure $\mathrm{MoNb}_{15} \mathrm{O}_{40} \mathrm{~F}$ se replace dans la série $B_{n m+1} \mathrm{O}_{3 n m-(n+m)+4}$ pour les valeurs: $m=3$ et $n=5$.

\section{Préparation et caractérisation}

L'oxyfluorure double $\mathrm{MoNb}_{15} \mathrm{O}_{40} \mathrm{~F}$ a été obtenu pur par synthèse directe à $1000^{\circ} \mathrm{C}$ en tube scellé de platine de quantités stoechiométriques d'oxydes de niobium $\mathrm{Nb}_{2} \mathrm{O}_{5}$, de molybdène $\mathrm{MoO}_{3}$ et d'oxyfluorure de niobium $\mathrm{NbO}_{2} \mathrm{~F}$ suivant l'équation de réaction:

$$
7 \mathrm{Nb}_{2} \mathrm{O}_{5}+\mathrm{MoO}_{3}+\mathrm{NbO}_{2} \mathrm{~F} \rightarrow \mathrm{MoNb}_{15} \mathrm{O}_{40} \mathrm{~F} .
$$

La réaction complète au bout de 12 heures de chauffe, donne un matériau homogène, blanc, bien cristallisé. Le spectre de poudre réalisé à l'aide d'une chambre Guinier-Hägg pour la radiation $\mathrm{Cu} K \alpha$ et calibré intérieurement avec du chlorure de potassium est donné au Tableau 1.

Tableau 1. Spectre de poudre de $\mathrm{MoNb}_{15} \mathrm{O}_{40} \mathrm{~F}(\mathrm{Cu} K \dot{\alpha})$

$\begin{array}{lccccc}I_{\mathrm{obs}} & \sin ^{2} \theta_{\mathrm{obs}} & \sin ^{2} \theta_{\text {calc }} & h & k & l \\ v w & 0,00176 & 0,00173 & 0 & 0 & 1 \\ w & 0,00484 & 0,00489 & 2 & 0 & \frac{1}{1} \\ v w & 0,00683 & 0,00692 & 0 & 0 & 2 \\ v w & 0,00740 & 0,00752 & 2 & 0 & 2 \\ v w & 0,01782 & 0,01776 & 2 & 0 & 2 \\ v w & 0,01948 & 0,01957 & 4 & 0 & \frac{2}{3} \\ m & 0,02301 & 0,02311 & 4 & 0 & \frac{3}{3} \\ w & 0,02898 & 0,02897 & 2 & 0 & 3 \\ s t & 0,04184 & 0,04186 & 1 & 1 & 0 \\ s t & 0,04221 & 0,04231 & 1 & 1 & 1 \\ v s t & 0,04389 & 0,04364 & 2 & 0 & 4 \\ w & 0,04405 & 6 & 0 & \frac{1}{3} \\ w & 0,04464 & 0,04487 & 1 & 1 & 1 \\ v s t & 0,04846 & 0,04847 & 6 & 0 & \frac{1}{4} \\ v w & 0,05119 & 0,05134 & 1 & 1 & 2 \\ m & 0,05246 & 0,05254 & 3 & 1 & \frac{2}{2} \\ & 0,05264 & 2 & 0 & 6\end{array}$

Tous les cristaux étudiés sont invariablement maclés comme le révèlent les clichés de Weissenberg. Cependant après avoir séparé les réseaux réciproques, qui s'interpénétraient, il a été possible de déterminer une maille monoclinique.

Les paramètres donnés au Tableau 2 avec les autres constantes cristallographiques permettent d'indexer parfaitement le spectre de poudre.

Tableau 2. Données cristallographiques de $\mathrm{MoNb}_{15} \mathrm{O}_{40} \mathrm{~F}$

$\begin{array}{lc}\text { Symétrie: } & \text { monoclinique } \\ \text { Paramètres: } & a=22,29 \pm 0,01 \AA \\ & b=3,831 \pm 0,002 \\ & c=20,27 \pm 0,01 \\ & \beta=114,00 \pm 0,05^{\circ} \\ \text { Groupe spatial } & C 2, C m \text { ou } C 2 / m \\ D_{m} & 4,48 \\ D_{x} & 4,51 \\ Z & 2\end{array}$

Une seule règle d'extinctions systématiques a été relevée: $h+k=2 n+1$ pour $h k l$, correspondant aux groupes spatiaux $C 2, C m$ ou $C 2 / m$.

\section{Détermination de la structure}

Les données cristallographiques pour la détermination de la structure ont été collectées à partir d'un cristal de dimensions $0,03 \mathrm{~mm} \times 0,02 \mathrm{~mm}$ à l'aide d'une chambre de Weissenberg munie d'un dispositif d'intégration (rayonnement utilisé: $K \alpha$ du cuivre).

La mesure des intensités a été faite visuellement par comparaison des taches de diffraction avec celles d'une échelle photographique préalablement étalonnée. Seule la correction par le facteur de Lorentz-polarisation a été effectuée.

Les facteurs de diffusion atomique pour $\mathrm{Nb}^{5+}$ sont ceux déterminés par Thomas \& Umeda (1957) et pour

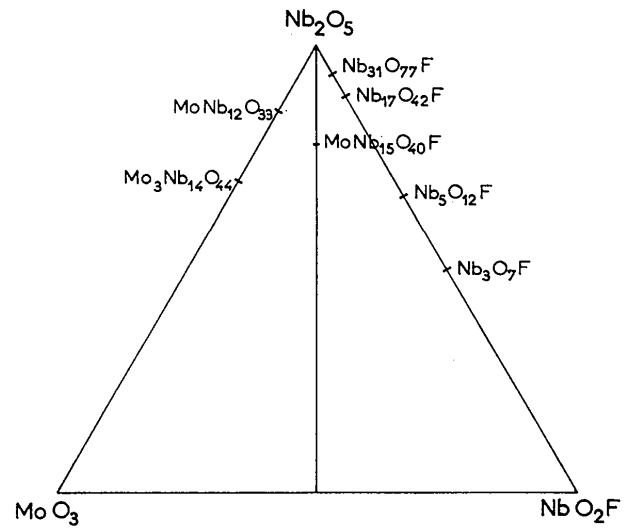

Fig. 1. Représentation géométrique du système $\mathrm{MoO}_{3}-\mathrm{Nb}_{2} \mathrm{O}_{5}-\mathrm{NbO}_{2} \mathrm{~F}$ 
$\mathrm{O}^{2-}$ ceux déterminés par Suzuki (1960); en raison des faibles différences existant entre leurs facteurs de diffusion, l'atome de molybdène a été traité comme un atome de niobium et l'atome de fluor comme un atome d'oxygène.

Les calculs ont été réalisés sur IBM 7090.

Une sous-maille simple orthorhombique présentant de grandes analogies avec la structure de type $\mathrm{ReO}_{3}$ a été reconnue dans le réseau réciproque; les relations vectorielles entre la vraie maille et la sous-maille ont été établies:

$$
\begin{aligned}
& a=5 a_{\mathrm{ReO}_{3}}+2 c_{\mathrm{ReO} 3} \\
& b=b_{\mathrm{ReO}_{3}} \\
& c=-3,5 a_{\mathrm{ReO}_{3}}+3,5 c_{\mathrm{ReO} 3} .
\end{aligned}
$$

A partir de ces relations une structure idéalisée a été obtenue à l'aide de méthodes précédemment développées par Andersson \& Wadsley (1962). Cette structure idéalisée présente quelques similitudes avec celle de $\mathrm{WNb}_{12} \mathrm{O}_{33}$; cette dernière est en effet constituée de blocs de type $\mathrm{ReO}_{3}$ de $3 \times 4$ octaèdres de côtés, et la structure qui en dérive en prenant des blocs de $3 \times 5$ octaèdres de côtés correspond à la composition $\mathrm{MoNb}_{15} \mathrm{O}_{40} \mathrm{~F}$. Des distorsions calculées à partir de $\mathrm{WNb}_{12} \mathrm{O}_{33}$ ont été introduites dans la structure idéalisée de $\mathrm{MoNb}_{15} \mathrm{O}_{40} \mathrm{~F}$. Le groupe spatial $C 2$ a été choisi.

Un premier calcul révèle un bon accord entre les 215 facteurs de structure observés et calculés dans les plans $h 0 l$ et $h 1 l$. Ceci confirme le choix du groupe spatial $C 2$. Une série de cycles d'affinement par la méthode des moindres carrés a permis d'abaisser le facteur de reliabilité jusqu'à la valeur $R=0,13$.

Les coordonnées réduites des divers atomes sont rassemblées au Tableau 3, les distances interatomiques métal-oxygène au Tableau 4 et la comparaison entre facteurs de structure observés et calculés au Tableau 5.

La projection de la structure suivant l'axe $O y$ est représentée à la Fig. 2.

Tableau 3. Coordonnées réduites des atomes

\begin{tabular}{lcccl}
\multicolumn{5}{c}{ Groupe spatial $C 2$} \\
Position & $x$ & $y$ & \multicolumn{1}{c}{$z$} \\
$\mathrm{Mo}$ & $2(a)$ & 0 & 0,250 & 0 \\
$\mathrm{Nb}(1)$ & $2(b)$ & $\frac{1}{2}$ & 0,000 & $\frac{1}{2}$ \\
$\mathrm{Nb}(2)$ & $4(c)$ & 0,3696 & 0,000 & 0,5749 \\
$\mathrm{Nb}(3)$ & $4(c)$ & 0,2371 & 0,000 & 0,3880 \\
$\mathrm{Nb}(4)$ & $4(c)$ & 0,3566 & 0,000 & 0,2940 \\
$\mathrm{Nb}(5)$ & $4(c)$ & 0,5023 & 0,000 & 0,2376 \\
$\mathrm{Nb}(6)$ & $4(c)$ & 0,0943 & 0,000 & 0,1804 \\
$\mathrm{Nb}(7)$ & $4(c)$ & 0,2326 & 0,000 & 0,1169 \\
$\mathrm{Nb}(8)$ & $4(c)$ & 0,3574 & 0,000 & 0,0286 \\
$\mathrm{~F}$ & $2(b)$ & $\frac{1}{2}$ & 0,500 & $\frac{1}{2}$ \\
$\mathrm{O}(1)$ & $4(c)$ & 0,292 & 0,000 & 0,603 \\
$\mathrm{O}(2)$ & $4(c)$ & 0,442 & 0,000 & 0,552 \\
$\mathrm{O}(3)$ & $4(c)$ & 0,293 & 0,000 & 0,469 \\
$\mathrm{O}(4)$ & $4(c)$ & 0,441 & 0,000 & 0,397 \\
$\mathrm{O}(5)$ & $4(c)$ & 0,563 & 0,000 & 0,330 \\
$\mathrm{O}(6)$ & $4(c)$ & 0,149 & 0,000 & 0,422 \\
$\mathrm{O}(7)$ & $4(c)$ & 0,292 & 0,000 & 0,335 \\
$\mathrm{O}(8)$ & $4(c)$ & 0,361 & 0,500 & 0,305 \\
$\mathrm{O}(9)$ & $4(c)$ & 0,434 & 0,000 & 0,273
\end{tabular}

Table 3 (suite)

$\begin{array}{lcccc} & \text { Position } & x & y & z \\ \text { O(10) } & 4(c) & 0,571 & 0,000 & 0,196 \\ \text { O(11) } & 4(c) & 0,165 & 0,000 & 0,289 \\ O(12) & 4(c) & 0,295 & 0,000 & 0,206 \\ O(13) & 4(c) & 0,438 & 0,000 & 0,139 \\ O(14) & 4(c) & 0,035 & 0,000 & 0,209 \\ O(15) & 4(c) & 0,165 & 0,000 & 0,145 \\ O(16) & 4(c) & 0,299 & 0,000 & 0,071 \\ O(17) & 4(c) & 0,065 & 0,500 & 0,011 \\ O(18) & 4(c) & 0,035 & 0,000 & 0,073 \\ O(19) & 4(c) & 0,156 & 0,000 & 0,011 \\ O(20) & 4(c) & 0,199 & 0,500 & 0,067\end{array}$

Tableau 4. Distances interatomiques (erreur $\pm 0,15 \AA)$ en $\AA$

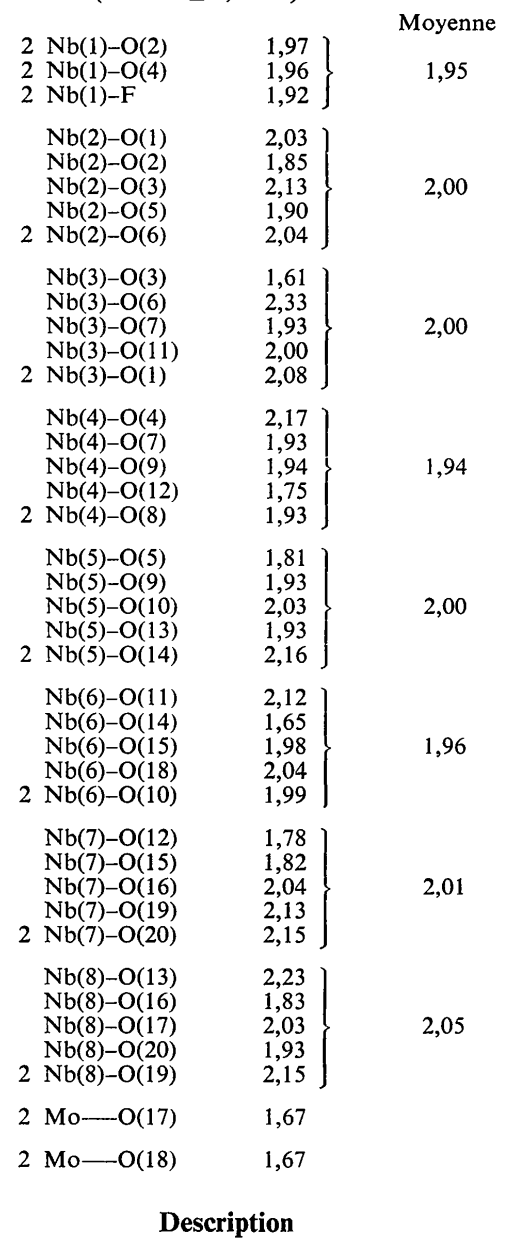

La structure de $\mathrm{MoNb}_{15} \mathrm{O}_{40} \mathrm{~F}$ schématisée par la Fig. 2 est constituée de blocs de type $\mathrm{ReO}_{3}$ de $3 \times 5 \times \infty$ octaèdres de côtés. Ces blocs sont reliés aux blocs similaires 
des différents niveaux par l'intermédiaire d'arêtes communes le long de l'axe $O y$; à la jonction de chaque groupe de quatre blocs se trouve un site tétraédrique. Ces blocs possèdent un centre de symétrie; l'octaèdre central est constitué par quatre atomes d'oxygène et deux atomes de fluor entourant un atome de niobium.
L'atome de molybdène Mo a été placé dans le site tétraédrique.

\section{Discussion}

La valeur moyenne des distances interatomiques $\mathrm{Nb}-\mathrm{O}$ dans $\mathrm{MoNb}_{15} \mathrm{O}_{40} \mathrm{~F}$, soit $1,99 \AA$, est comparable aux

Tableau 5. Comparaison des facteurs de structures observés et calculées
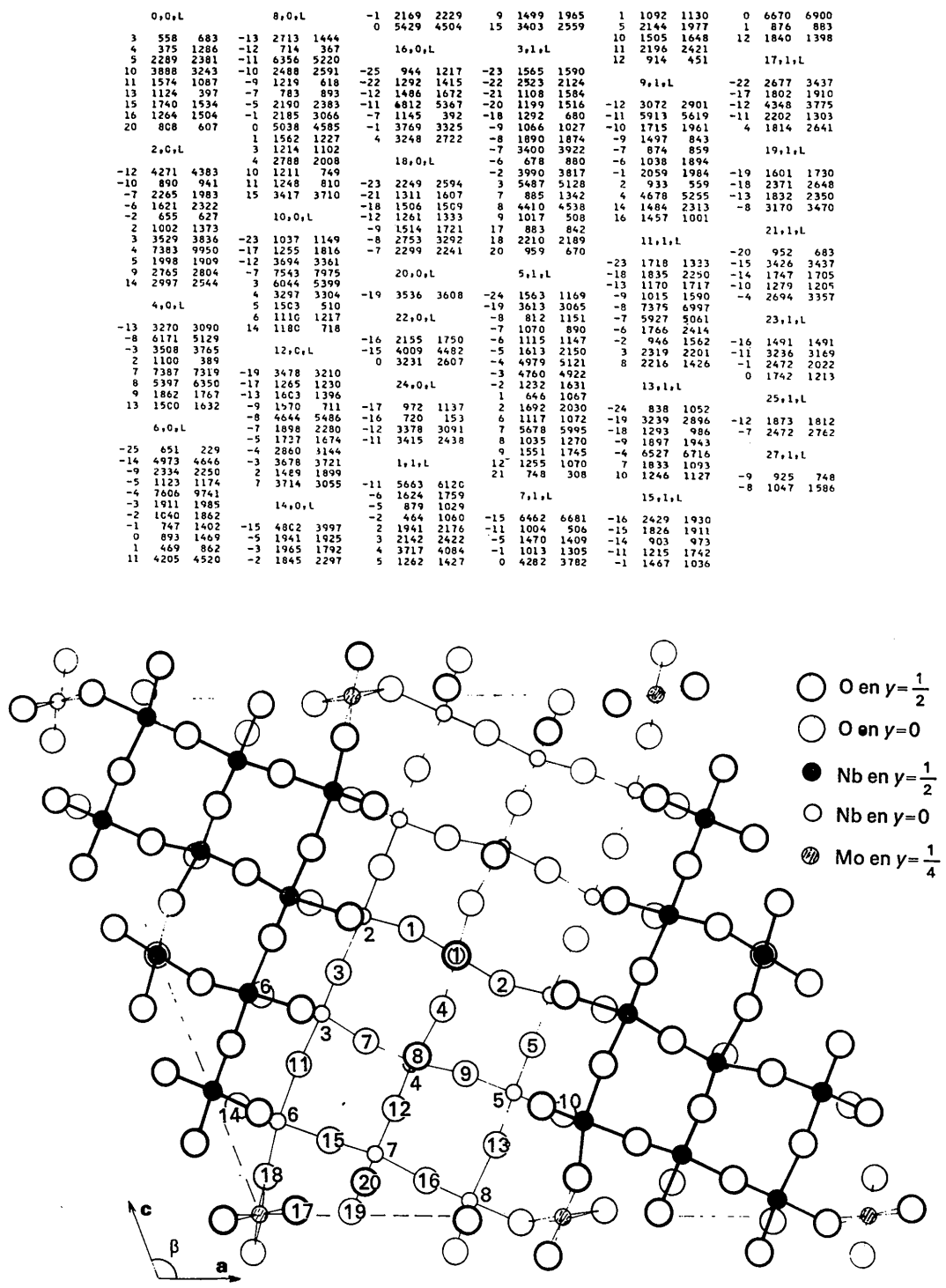

Fig. 2. Projection de la structure de $\mathrm{MoNb}_{15} \mathrm{O}_{40} \mathrm{~F}$ sur le plan (010). 


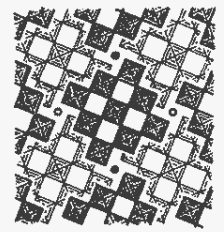

$\mathrm{PNb}_{9} \mathrm{O}_{25}$

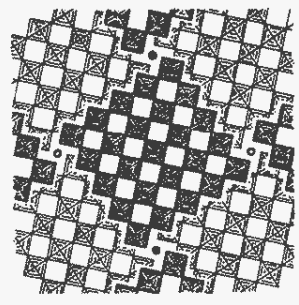

$\mathrm{W}_{8} \mathrm{Nb}_{18} \mathrm{O}_{69}$

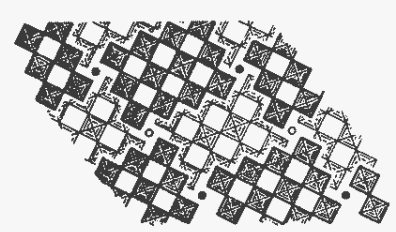

$\mathrm{WNb}_{12} \mathrm{O}_{33}$

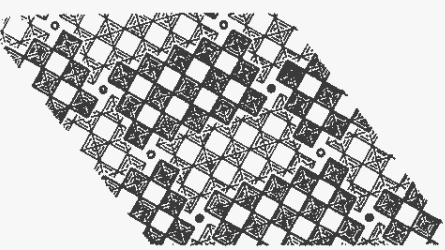

$\mathrm{MoNb}{ }_{15} \mathrm{O}_{40} \mathrm{~F}$

Fig. 3. Structures idéalisées de $\mathrm{PNb}_{9} \mathrm{O}_{25}$ (blocs $3 \times 3 \times \infty$ ), $\mathrm{WNb}_{12} \mathrm{O}_{33}$ (blocs $3 \times 4 \times \infty$ ), MoNb $15 \mathrm{O}_{40} \mathrm{~F}$ (blocs $3 \times 5 \times \infty$ ) et $\mathrm{W}_{8} \mathrm{Nb}_{18} \mathrm{O}_{69}$ (blocs $\left.5 \times 5 \times \infty\right)$. Les cercles représentent les atomes en site tétraédrique.

distances $\mathrm{Nb}-\mathrm{O}$ observées dans $\mathrm{H}-\mathrm{Nb}_{2} \mathrm{O}_{5}(1,99 \AA)$ par Gatehouse \& Wadsley (1964) ou dans $\mathrm{N}-\mathrm{Nb}_{2} \mathrm{O}_{5}$ (2,01 A) par Andersson (1967).

La moyenne des distances interatomiques Mo-O $(1,67 \AA)$ correspondant à l'environnement tétraédrique est en bon accord avec les résultats précédemment obtenus pour ce type de sites dans $\mathrm{H}-\mathrm{Nb}_{2} \mathrm{O}_{5}(\mathrm{Nb}-\mathrm{O}=$ $1,67 \AA)$.

Reprenant la formulation structurale d'Andersson $(1965 b), \mathrm{MoNb}_{15} \mathrm{O}_{40} \mathrm{~F}$ peut être représenté par le symbole structural:

$$
\begin{array}{lll}
\mathrm{O}=\mathrm{O}-\mathrm{O}-\mathrm{O} \rightarrow n \\
\mid & \| \\
\mathrm{O}-T & -\mathrm{O} \rightarrow n \\
\| & \mid & \mid \\
\mathrm{O}-\mathrm{O}=\mathrm{O} \rightarrow n & \mathrm{O} \rightarrow n \\
\downarrow & \downarrow & \downarrow \\
m & m & m
\end{array}
$$

avec le molybdène dans le site tétraédrique $T$, la dimension des blocs de type $\mathrm{ReO}_{3}$ étant indiquée par $m$ et $n\left(m=3\right.$ et $n=5$ dans $\left.\mathrm{MoNb}_{15} \mathrm{O}_{40} \mathrm{~F}\right)$. La structure de $\mathrm{MoNb}_{15} \mathrm{O}_{40} \mathrm{~F}$ apparaît extrêmement originale parmi les composés appartenant à ce type structural; en effet on connaissait:

\begin{tabular}{lrl} 
& $m \times n$ & \multicolumn{1}{c}{ Symétrie } \\
$\mathrm{PNb}_{9} \mathrm{O}_{25}$ (Fig. 3) & $3 \times 3$ & Quadratique \\
$\mathrm{WNb}_{12} \mathrm{O}_{33}$ (Fig. 3) & $3 \times 4$ & Monoclinique \\
$\mathrm{W}_{3} \mathrm{Nb}_{14} \mathrm{O}_{44}$ & $4 \times 4$ & Quadratique \\
$\mathrm{W}_{5} \mathrm{Nb}_{16} \mathrm{O}_{55}$ & $4 \times 5$ & Monoclinique \\
$\mathrm{W}_{8} \mathrm{Nb}_{18} \mathrm{O}_{69}$ (Fig. 3) & $5 \times 5$ & Quadratique
\end{tabular}

mais dans ces structures la différence maximum entre longueur et largeur des blocs de type $\mathrm{ReO}_{3}$ était tout au plus d'une unité. $\mathrm{MoNb}_{15} \mathrm{O}_{40} \mathrm{~F}$ est le premier exemple où $n-m>1(n-m=2)$.

L'impossibilité d'obtenir un composé isostructural en remplaçant $\mathrm{NbO}_{2} \mathrm{~F}$ par $\mathrm{MoO}_{3}$ ou $\mathrm{WO}_{3}$ nous incite à accorder au fluor un rôle non négligeable dans la stabilisation de cette structure.

J. Galy voudrait remercier le Professeur Arne Magnéli, Institut de Chimie Minérale, Université de Stockholm, Suède, pour son hospitalité durant le stage qu'il a effectué à Stockholm, stage au cours duquel ce tra. vail a été réalisé.

\section{Références}

Andersson, S. (1964). Acta Chem. Scand. 18, 2339. Andersson, S. (1965a). Acta Chem. Scand. 19, 1401. ANDERSSON, S. (1965b). Bull. Soc. chim. France, 1088. ANDERSSON, S. (1967). Z. anorg. allg. Chem. 351, 106. Andersson, S. \& Wadsley, A. D. (1962). Acta Cryst. 15, 194.

Andersson, S. \& Åström, A. (1964). Acta Chem. Scand. $18,2233$.

Gatehouse, B. M. \& Wadsley, A. D. (1964), Acta Cryst. $17,1545$.

Roth, R. S. \& Wadsley, A. D. (1965). Acta Cryst. 19, 26, $32,38,42$.

Suzuki, T. (1960). Acta Cryst. 13, 279.

Thomas, L. H. \& UMEDA, K. (1957). J. Chem. Phys. 26, 293. Åström, A. (1966). Acta Chem. Scand. 20, 969. 